\title{
Impulsive Control of Memristive Chaotic Systems with Impulsive Time Window
}

\author{
FuLi Chen, ${ }^{1}$ Hui Wang, ${ }^{1,2}$ and Chuandong $\mathrm{Li}^{2}$ \\ ${ }^{1}$ College of Mathematics Science, Chongqing Normal University, Chongqing 401331, China \\ ${ }^{2}$ College of Electronic and Information Engineering, Southwest University, Chongqing 400715, China \\ Correspondence should be addressed to Hui Wang; huikittycat@gmail.com
}

Received 29 December 2014; Accepted 2 March 2015

Academic Editor: Antonino Laudani

Copyright (c) $2015 \mathrm{FuLi}$ Chen et al. This is an open access article distributed under the Creative Commons Attribution License, which permits unrestricted use, distribution, and reproduction in any medium, provided the original work is properly cited.

\begin{abstract}
The problem of impulsive control for memristor-based chaotic circuit systems with impulsive time windows is investigated. Based on comparison principle, several novel criteria which guarantee the asymptotic stabilization of the memristor-based chaotic circuit systems are obtained. In comparison with previous results, the present results are easily verified. Numerical simulations are given to further illustrate the effectiveness of the theoretical results.
\end{abstract}

\section{Introduction}

In 1971 [1], Chua presented the logical and scientific basis for the existence of a new two-terminal circuit element named the memristor which has every right to as basic as the three classical circuit elements already in existence, namely, the resistor, inductor, and capacitor. However, this important presentation has not aroused any attention until researchers at Hewlett-Packard Laboratories published a paper announcing the invention of the memristor in 2008 [2, 3]. Memristor has been widely investigated for its immense potential applications in different areas such as brain emulation, associative memory, and pattern recognition. This new circuit element of memristor shares many properties of resistor and shares the same unit of measurement. Therefore, memristor is nonlinear and shows many special properties. On the other hand, memristive chaotic systems made of hybrid complementary metal-oxide-semiconductors have a very wide range of uses in bioinspired engineering. Analysis and synthesis of memristive chaotic systems are very attractive for neuromorphic systems. Moreover, the development of high-performance memristive chaotic systems would benefit a number of important applications in neural learning circuits, new classes of artificial neural systems, and so forth.

We also note that impulsive control has been widely used to stabilize and synchronize chaotic systems [4-11] and also employed in many fields, such as ecosystems management [12], orbital transfer of satellite [13], and optimal control of economic systems [14]. Impulsive control can provide an efficient method for some case in which the systems cannot endure continuous disturbance. Most of the researchers are dealing with impulsive differential equations with fixed time impulses $[8,15,16]$ or with variable time impulse [17]. If we put impulses in a fixed time for a system, then we get an impulsive system with fixed time impulses. However, it is not easy to ensure that the input is exact according to the fixed time points. If the occurrence of impulses is determined by the statement of the system, then it is a system with variable time impulses, and if the system is known, that means it is hard for us to know the exact statement of the system, the process of calculating the time sequences of impulses may bring some errors, and then the impulse may occur in an unexpected time. Although there are many results concerning the impulsive control for nonlinear systems [18], the control problem of memristive systems with impulsive effects has received little attention. Moreover, in many existing results regarding impulsive effects occurring at a fixed moment, such an assumption is conservative, as systems may be subjected to instantaneous disturbances at any time instead of only at the fixed points. Therefore, it is of great importance to consider a more general case where impulsive effects can exist at a random moment of 


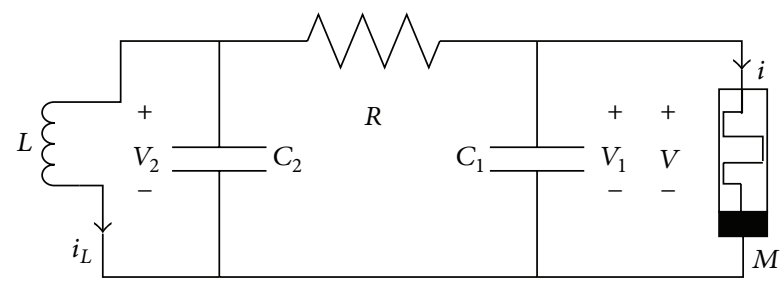

Figure 1: The memristor chaotic circuit [19].

a determined time interval. However, to the best of our knowledge, the impulsive control problem for memristive chaotic system with impulsive time window has aroused little attention due to its mathematical complexity. Therefore, the main objective of this paper is to shorten such a gap by launching a study on the impulsive control problem for memristive chaotic system with impulsive time window.

In this paper, we are concerned with the asymptotical stabilization problem for a class of memristive chaotic systems with impulsive time window. Based on comparison principle, we will propose several novel criteria for the stabilization of memristive chaotic system with impulsive time window. The main contributions of this paper can be highlighted as follows: (1) a new memristive chaotic system model is established to consider impulsive time window, where impulsive effects can exist at a random moment of a determined time interval; (2) by using comparison principle, sufficient criteria are derived to ensure that memristive chaotic system with impulsive time window is asymptotically stable; (3) an impulsive time window, memristive for modeling the chaotic systems simultaneously, renders more practical significance of current research.

The remainder of the paper is organized as follows. The considered model of a general memristive chaotic system with impulsive time window is presented in Section 2. Some necessary definitions and lemmas are given in Section 2. And the impulsive control problem for memristive system is also studied in Section 3. Then, in Section 4, a simulation example is presented to show effectiveness and feasibility of the new results. Conclusions are drawn in Section 5.

\section{Model Description and Preliminaries}

Consider the memristor-based chaotic circuit of [19] shown in Figure 1. The equations of the memristor-based chaotic in Figure 1 can be described by

$$
\begin{aligned}
& \frac{d v_{1}(t)}{d t}=\frac{1}{c_{1}}\left(\frac{v_{2}(t)-v_{1}(t)}{R}-i(t)\right), \\
& \frac{d v_{2}(t)}{d t}=\frac{1}{c_{2}}\left(\frac{v_{1}(t)-v_{2}(t)}{R}-i_{L}(t)\right), \\
& \frac{d i_{L}(t)}{d t}=\frac{v_{2}(t)}{L} \\
& \frac{d \varphi(t)}{d t}=v_{1}(t),
\end{aligned}
$$

where

$$
i(t)=w(\varphi(t)) v_{1}(t)=\frac{d q}{d \varphi} v_{1}(t) .
$$

Let $v_{1}=x_{1}, v_{2}=x_{2}, i_{L}=x_{3}, \varphi=x_{4}, a=1 / c_{1}, b=1 / L$, $R=1$, and $c_{2}=1$; then system (1) can be rewritten as

$$
\begin{aligned}
& \dot{x}_{1}=a\left(x_{2}-x_{1}-w(\varphi) x_{1}\right), \\
& \dot{x}_{2}=x_{1}-x_{2}-x_{3}, \\
& \dot{x}_{3}=b x_{2}, \\
& \dot{x}_{4}=x_{1},
\end{aligned}
$$

where

$$
w(\varphi)=\frac{d q}{d \varphi}=\alpha+3 \beta \varphi^{2}
$$

Usually, in order to obtain the chaos generation, we settled the realistic parameter values which yield chaotic dynamic as $R=2 \mathrm{k} \Omega, L=14.5 \mathrm{H}, c_{1}=5.6 \mathrm{uF}$, and $c_{2}=78 \mathrm{uF}$ potentiometer with parameters $\alpha=-0.663 \times 10^{-3}, \beta=-0.004 \times$ $10^{-3}$ in (4). Choosing the initial conditions $\left(v_{1}(0), v_{2}(0)\right.$, $\left.i_{L}(0), \varphi(0)\right)=(0.1254,0.1402,0.0921,0.0007)$, the attractor generated by means of numerical integration is illustrated in Figure 2.

Similar to the method presented in [20], system (3) can be rewritten as

$$
\dot{X}=A X+\phi(X),
$$

where

$$
\begin{gathered}
X^{T}=\left(x_{1}, x_{2}, x_{3}, x_{4}\right), \quad A=\left(\begin{array}{cccc}
-a & a & 0 & 0 \\
1 & -1 & -1 & 0 \\
0 & b & 0 & 0 \\
1 & 0 & 0 & 0
\end{array}\right), \\
\phi(X)=\left(\begin{array}{ccc}
-a w(\varphi) x_{1} \\
0 \\
0 & \\
0 &
\end{array}\right), \\
\phi^{T} \phi=a^{2} w^{2}(\varphi) x_{1}^{2}=X^{T} J X, \\
J=\left(\begin{array}{cccc}
a^{2} w^{2}(\varphi) & 0 & 0 & 0 \\
0 & 0 & 0 & 0 \\
0 & 0 & 0 & 0 \\
0 & 0 & 0 & 0
\end{array}\right) .
\end{gathered}
$$

By introducing the impulsive effects into the model [20], we obtain the following memristive chaotic system with impulsive effects:

$$
\begin{aligned}
& \dot{X}=A X+\phi(X) \quad t \neq \tau_{k}, \tau_{k} \in\left[\tau_{k}^{l}, \tau_{k}^{r}\right), \\
& X\left(\tau_{k}^{+}\right)=B X\left(\tau_{k}^{l}\right) \quad t=\tau_{k}, \\
& X\left(\tau_{0}^{+}\right)=X_{0} \quad k \in N,
\end{aligned}
$$



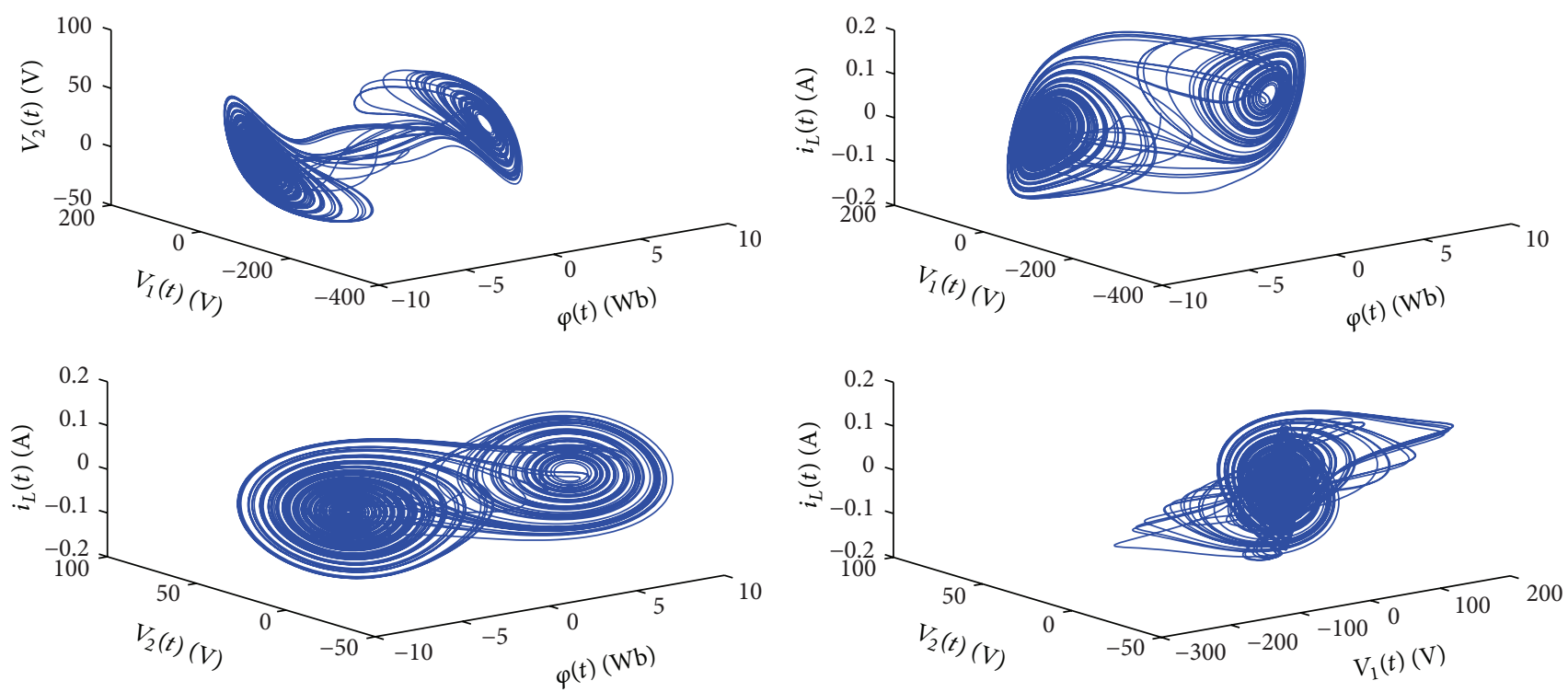

FIGURE 2: Simulation results by MATLAB, the chaotic attractor codified by (1).

where $\tau_{k}$ denote the moments when impulsive control occurs. $\left[\tau_{k}^{l}, \tau_{k}^{r}\right)$ are impulsive time windows. We assume that $\tau_{k}^{r}-\tau_{k}^{l}=$ $\delta$ (for all $k=0,1,2, \ldots$ ), where $\delta>0$ is a constant and $\tau_{k+1}^{l}$ $\tau_{k}^{r}=d$ (for all $k=0,1,2, \ldots$ ), where $d>0$ is a constant; that is, $\tau_{k}^{l}=\tau_{0}^{l}+k(\delta+d)$ and $\tau_{k}^{r}=\tau_{0}^{l}+k(\delta+d)+d, \tau_{0}^{l} \leq \tau_{0}<$ $\tau_{0}^{r} \leq \tau_{1}^{l}<\tau_{1} \leq \tau_{1}^{r} \leq \cdots \leq \tau_{k}^{l}<\tau_{k} \leq \tau_{k}^{r}, \lim _{k \rightarrow \infty} \tau_{k}=\infty$. We can see that a memristor-based chaotic circuit system with impulsive time windows is more difficult to research than the system with fixed-time impulse.

The following definitions and lemmas are presented for the derivation of the main results.

Definition 1 (see [21]). Let $V: R_{+} \times R^{n} \rightarrow R_{+}$; then $V$ is said to belong to class $v_{0}$ if

(1) $V$ is continuous in $\left(\tau_{k-1}, \tau_{k}\right] \times R^{n}$ and, for each $x \in R^{n}$,

$$
\lim _{(t, y) \rightarrow\left(\tau_{k}^{+}, x\right)} V(t, y)=V\left(\tau_{k}^{+}, x\right)
$$

exist, for any $k=1,2, \ldots$.

(2) $V$ is locally Lipschitzian in $x$ and $V(t, 0)=0$ for all $t \in R^{+}$.

Definition 2. Let $V \in V_{0}$ and assume that

$$
\begin{aligned}
& D^{+} V(t, x) \leq g(t, V(t, x)) \quad t \neq \tau_{k}, \tau_{k} \in\left[\tau_{k}^{l}, \tau_{k}^{r}\right), \\
& V\left(\tau_{k}, x\left(\tau_{k}^{+}\right)\right) \leq \psi_{k}\left(V\left(\tau_{k}^{l}, x\left(\tau_{k}^{l}\right)\right), V\left(\tau_{k}^{r}, x\left(\tau_{k}^{r}\right)\right)\right),
\end{aligned}
$$

where $g: R^{+} \times R \rightarrow R$ is continuous and $\psi_{k}$ is nondecreasing. Then the following system:

$$
\begin{aligned}
& \dot{\omega}=g(t, \omega) \quad t \neq \tau_{k}, \tau_{k} \in\left[\tau_{k}^{l}, \tau_{k}^{r}\right), \\
& \omega\left(\tau_{k}^{+}\right)=\psi_{k}\left(\omega\left(\tau_{k}^{l}\right), \omega\left(\tau_{k}^{r}\right)\right), \\
& \omega\left(\tau_{0}^{+}\right)=\omega_{0} \geq 0
\end{aligned}
$$

is called the comparison system of (7).
Lemma 3 (see [22]). Given any real matrices $\sum_{1}, \sum_{2}$ of appropriate dimensions and a scalar $\varepsilon>0$, then the following inequality holds:

$$
\sum_{1}^{T} \sum_{2}+\sum_{2}^{T} \sum_{1} \leq \varepsilon \sum_{1}^{T} \sum_{1}+\mathcal{E}^{-1} \sum_{2}^{T} \sum_{2}
$$

Definition 4. Assume that

(1) $f(t, 0)=0, g(t, 0)=0$, and $\psi_{k}(0,0)=0$ and $U(k, 0)$ for all $k$;

(2) $V: R_{+} \times S_{\rho} \rightarrow R_{+}, \rho>0, V \in V_{0}, D^{+} V(t, x) \leq$ $g(t, V(t, x)), t \neq \tau_{k}$

(3) there exists a $\rho_{0}$ such that $x\left(\tau_{k}^{l}\right) \in S_{\rho_{0}}$, implying that $x\left(\tau_{k}^{+}\right) \in S_{\rho}$ for all $k$, and $V\left(\tau_{k}, x\left(\tau_{k}^{+}\right)\right) \leq \psi\left(V\left(\tau_{k}^{l}\right.\right.$, $\left.\left.x\left(\tau_{k}^{l}\right)\right), V\left(\tau_{k}^{r}, x\left(\tau_{k}^{r}\right)\right)\right), x\left(\tau_{k}^{l}\right) \in S_{\rho_{0}} ;$

(4) $\beta(x) \leq V(t, x) \leq \alpha(x)$ on $R_{+} \times S_{\rho}$, where $\alpha, \beta \in k$.

Then the stability properties of the trivial solution of comparison system (10) imply the corresponding stability properties of the trivial solution of (7).

Lemma 5. Consider the following system:

$$
\begin{aligned}
& \dot{\omega}=\dot{\lambda}(t) \omega \quad t \neq \tau_{k}, \tau_{k} \in\left[\tau_{k}^{l}, \tau_{k}^{r}\right), \\
& \omega\left(\tau_{k}^{+}\right)=d_{k} \omega\left(\tau_{k}^{l}\right), \\
& \omega\left(\tau_{0}^{+}\right)=\omega_{0} \geq 0 \quad k \in N,
\end{aligned}
$$

where $\lambda \in C^{1}\left[R_{+}, R_{+}\right], \dot{\lambda}(t) \geq 0$, for all $k \in N$; then the trivial solution of (7) is asymptotically stable if

$$
\lambda\left(\tau_{k+1}^{r}\right)-\lambda\left(\tau_{k}^{l}\right)+\ln \left(\gamma d_{k}\right) \leq 0
$$

for all $k \in N$, where $\gamma>1$ is satisfied. 
Proof. Let $\omega\left(t, \tau_{0}, \omega_{0}\right)$ be any solution of the following comparison system (12). Then we have

$$
\begin{aligned}
& \omega\left(t, \tau_{0}, \omega_{0}\right) \\
& \quad=\omega_{0} e^{\lambda(t)-\lambda\left(\tau_{0}\right)} \prod_{i=1}^{k} d_{i} e^{-\lambda\left(\tau_{i}\right)+\lambda\left(\tau_{i}^{l}\right)}, \quad t \in\left[\tau_{k}, \tau_{k+1}\right) .
\end{aligned}
$$

From $\lambda\left(\tau_{k+1}^{r}\right)-\lambda\left(\tau_{k}^{l}\right)+\ln \left(\gamma d_{k}\right) \leq 0$ we know that

$$
\begin{aligned}
& \lambda\left(\tau_{k+1}^{r}\right)-2 \lambda\left(\tau_{k}^{l}\right)+\lambda\left(\tau_{k}^{l}\right)+\ln \left(\gamma d_{k}\right) \leq 0, \\
& \lambda\left(\tau_{k+1}\right)-2 \lambda\left(\tau_{k}\right)+\lambda\left(\tau_{k}^{l}\right)+\ln \left(\gamma d_{k}\right) \leq 0 .
\end{aligned}
$$

So $d_{k} e^{\lambda\left(\tau_{k+1}\right)-2 \lambda\left(\tau_{k}\right)+\lambda\left(\tau_{k}^{l}\right)} \leq 1 / \gamma$, from (14) we know that, for $t \in\left[\tau_{k}, \tau_{k+1}\right)$,

$$
\begin{aligned}
\omega\left(t, \tau_{0}, \omega_{0}\right) & =\omega_{0} e^{\lambda(t)-\lambda\left(\tau_{0}\right)} \prod_{i=1}^{k} d_{i} e^{-\lambda\left(\tau_{i}\right)+\lambda\left(\tau_{i}^{l}\right)} \\
& \leq \omega_{0} e^{\lambda\left(\tau_{k+1}\right)-\lambda\left(\tau_{0}\right)} \prod_{i=1}^{k} d_{i} e^{-\lambda\left(\tau_{i}\right)+\lambda\left(\tau_{i}^{l}\right)} \\
& =\omega_{0}\left(\prod_{i=1}^{k} d_{i} e^{\lambda\left(\tau_{i+1}\right)-2 \lambda\left(\tau_{i}\right)+\lambda\left(\tau_{i}^{l}\right)}\right) e^{\lambda\left(\tau_{1}\right)-\lambda\left(\tau_{0}\right)} \\
& =\frac{\omega_{0}}{\gamma^{k}} e^{\lambda\left(\tau_{1}\right)-\lambda\left(\tau_{0}\right)}, \quad t \in\left[\tau_{k}, \tau_{k+1}\right) .
\end{aligned}
$$

Hence $\lim _{t \rightarrow \infty} \omega\left(t, \tau_{0}, \omega_{0}\right)=0$. We thus proved that the trivial solution of system (12) is asymptotically stable. Following Lemma 5 we know that the origin of system (7) is asymptotically stable.

\section{Main Results}

In this section, the problem of impulsive control for memristive chaotic system with impulsive time window is studied. We will find the impulsive time window $\left[\tau_{k}^{l}, \tau_{k}^{r}\right)$ such that the memristive chaotic system (7) is impulsively asymptotically stable. To do so, we give the following theorem and corollary.

Theorem 6. The matrix $P$ is symmetric and positive definite; let $d_{1}>0, d_{2}>0$ be the smallest and the largest eigenvalues of $P$; let $Q=A^{T} P+P A$, where $A^{T}$ is the transpose of $A$, and $d_{3}$ is the largest eigenvalue of $P^{-1} Q, d_{4}$ is the largest eigenvalue of the matrix $P^{-1} B^{T} P B$, respectively, and $\delta=\tau_{k}^{l}-\tau_{k}^{r}>0$, $d=\tau_{k+1}^{l}-\tau_{k}^{r}>0 ; \phi(X)$ is continuous and $\|\phi(X)\| \leq L\|X\|$ with $L>0$ being a constant. Then the origin of memristorbased chaotic circuit system with impulsive time windows (7) is asymptotically stable if there exists $a \xi>1$ such that $0 \leq$ $d_{3}+2 L\left(d_{2} / d_{1}\right) \leq-\ln \left(\xi d_{4} /(2 \delta+d)\right)$.

Proof. Let the Lyapunov function be in the form of $V(t, X)=$ $X^{T} P X$.
For $t \neq \tau_{k}$, we have

$$
\begin{aligned}
D^{+} V(t, X) & =\dot{X}^{T} P X+X^{T} P \dot{X} \\
& =(A X+\phi(X))^{T} P X+X^{T} P(A X+\phi(X)) \\
& =X^{T} A^{T} P X+\phi^{T}(X) P X+X^{T} P A X+X^{T} P \phi(X) \\
& =X^{T}\left(A^{T} P+P A\right) X+\phi^{T}(X) P X+X^{T} P \phi(X) \\
& \leq\left(d_{3}+2 L \frac{d_{2}}{d_{1}}\right) V(t, x) .
\end{aligned}
$$

Hence the second condition of Definition 4 is satisfied with $g(t, \omega)=\left(d_{3}+2 L\left(d_{2} / d_{1}\right)\right) \omega$.

Since $\left\|X\left(\tau_{k}^{+}\right)\right\|=\left\|B X\left(\tau_{k}^{l}\right)\right\| \leq\|B\|\left\|X\left(\tau_{k}^{l}\right)\right\|$ and $\|B\|$ is finite, there exists a $\rho_{0}>0$ and $X\left(\tau_{k}^{l}\right) \in S_{\rho_{0}}$, such that $X\left(\tau_{k}^{+}\right) \in S_{\rho}$.

For $t=\tau_{k}$, we have

$$
\begin{aligned}
V\left(\tau_{k}, X\left(\tau_{k}^{+}\right)\right) & =\left(B X\left(\tau_{k}^{l}\right)\right)^{T} P\left(B X\left(\tau_{k}^{l}\right)\right) \\
& =\left(X\left(\tau_{k}^{l}\right)\right)^{T}\left(B^{T} P B\right) X\left(\tau_{k}^{l}\right) \\
& \leq d_{4} V\left(\tau_{k}^{l}, X\left(\tau_{k}^{l}\right)\right) .
\end{aligned}
$$

Hence condition 3 of Definition 4 is satisfied with $\psi_{k}\left(\omega\left(\tau_{k}^{l}\right), \omega\left(\tau_{k}^{r}\right)\right)=d_{4} \omega\left(\tau_{k}^{l}\right)$. And we have $d_{1}\|X\|^{2} \leq V(X) \leq$ $d_{2}\|X\|^{2}$.

Therefore, condition 4 of Definition 4 is also satisfied with $\beta(x)=d_{1} x$ and $\alpha(x)=d_{2} x$. It follows from Definition 4 that the asymptotic stability of the memristor-based chaotic circuit system with impulsive time windows (7) is implied by that of the comparison system:

$$
\begin{aligned}
& \dot{\omega}=\left(d_{3}+2 L \frac{d_{2}}{d_{1}}\right) \omega t \neq \tau_{k}, \tau_{k} \in\left[\tau_{k}^{l}, \tau_{k}^{r}\right), k \in N, \\
& \omega\left(\tau_{k}^{+}\right)=d_{4} \omega\left(\tau_{k}^{l}\right), \\
& \omega\left(\tau_{0}^{+}\right)=\omega_{0}>0 .
\end{aligned}
$$

It follows from Lemma 5 that if

$$
\int_{\tau_{k}^{-}}^{\tau_{k+1}^{+}}\left(d_{3}+2 L \frac{d_{2}}{d_{1}}\right) d t+\ln \left(\xi d_{4}\right) \leq 0 \quad \xi>1,
$$

that is,

$$
\left(d_{3}+2 L \frac{d_{2}}{d_{1}}\right)\left(\tau_{k+1}^{+}-\tau_{k}^{-}\right) \leq-\ln \xi d_{4} \quad \xi>1,
$$

is satisfied, then the origin of (7) is asymptotically stable.

Remark 7. Theorem 6 gives the sufficient conditions for the asymptotic stability for controlling the systems to the origin. Without loss of generality, let the matrix $P=E$; then we get the following corollary. 
Corollary 8. Let $d_{1}$ be the largest eigenvalue of $B^{T} B$ and $q=$ $\|J\|$, let $d_{2}$ be the largest eigenvalue of $A^{T}+A$, and let impulses be equidistant with an interval $\delta>0$. If

$$
0<\left(d_{2}+\varepsilon q+\varepsilon^{-1}\right) \leq-\frac{1}{2 \delta+d} \ln \xi d_{1}
$$

then the origin of impulsively controlled memristor-based chaotic circuit system with impulsive time windows (7) is asymptotically stable.

Proof. Let the Lyapunov function be in the form of $V(t, X)=$ $X^{T} X$.

For $t \neq \tau_{k}$, from Lemma 3, we have that

$$
\begin{aligned}
D^{+} V(t, X) & =\dot{X}^{T} X+X^{T} \dot{X} \\
& =X^{T} A X+X^{T} A^{T} X+X^{T} \phi(X)+\phi^{T}(X) X \\
& =X^{T}\left(A+A^{T}\right) X+X^{T} \phi(X)+\phi^{T}(X) X \\
& \leq\left(d_{2}+\varepsilon q+\varepsilon^{-1}\right) V(t, X) .
\end{aligned}
$$

Hence the second condition of Definition 4 is satisfied with $g(t, \omega)=\left(d_{2}+\varepsilon q+\varepsilon^{-1}\right) \omega$.

Since $\left\|x\left(\tau_{k}^{+}\right)\right\|=\left\|B x\left(\tau_{k}^{l}\right)\right\| \leq\|B\|\left\|x\left(\tau_{k}^{l}\right)\right\|$ and $\|B\|$ is finite, there exists a $\rho_{0}>0$ and $x\left(\tau_{k}^{l}\right) \in S_{\rho_{0}}$, such that $x\left(\tau_{k}^{+}\right) \in S_{\rho}$.

For $t=\tau_{k}$, we have

$$
\begin{aligned}
V\left(\tau_{k}, x\left(\tau_{k}^{+}\right)\right) & =\left(B x\left(\tau_{k}^{l}\right)\right)^{T}\left(B x\left(\tau_{k}^{l}\right)\right) \\
& =x\left(\tau_{k}^{l}\right)^{T} B^{T} B x\left(\tau_{k}^{l}\right) \\
& \leq d_{1} V\left(\tau_{k}^{l}, x\left(\tau_{k}^{l}\right)\right) .
\end{aligned}
$$

Hence the third condition of Definition 4 is satisfied with $\psi_{k}\left(\omega\left(\tau_{k}^{l}\right), \omega\left(\tau_{k}^{r}\right)\right)=d_{1} \omega\left(\tau_{k}^{l}\right)$; the fourth condition of Definition 4 is also satisfied. It follows from Definition 4 that the asymptotic stability of the memristor-based chaotic circuit system with impulsive time windows (7) is implied by that of the comparison system:

$$
\begin{aligned}
& \dot{\omega}=\left(d_{2}+\varepsilon q+\varepsilon^{-1}\right) \omega \quad t \neq \tau_{k}, \tau_{k} \in\left[\tau_{k}^{l}, \tau_{k}^{r}\right), \\
& \omega\left(\tau_{k}^{+}\right)=d_{1} \omega\left(\tau_{k}^{l}\right), \\
& \omega\left(\tau_{0}^{+}\right)=\omega_{0} \geq 0 \quad k \in N .
\end{aligned}
$$

Since $0<\left(d_{2}+\varepsilon q+\varepsilon^{-1}\right) \leq-(1 /(2 \delta+d)) \ln \xi d_{1}$, we have

$$
\int_{\tau_{k}^{-}}^{\tau_{k+1}^{+}}\left(d_{2}+\varepsilon q+\varepsilon^{-1}\right) d t+\ln \left(\xi d_{1}\right) \leq 0 \quad \forall k \in N .
$$

It follows from Lemma 5 that (12) is asymptotically stable. And from Definition 4 we conclude that the trivial solution of (7) is asymptotically stable.

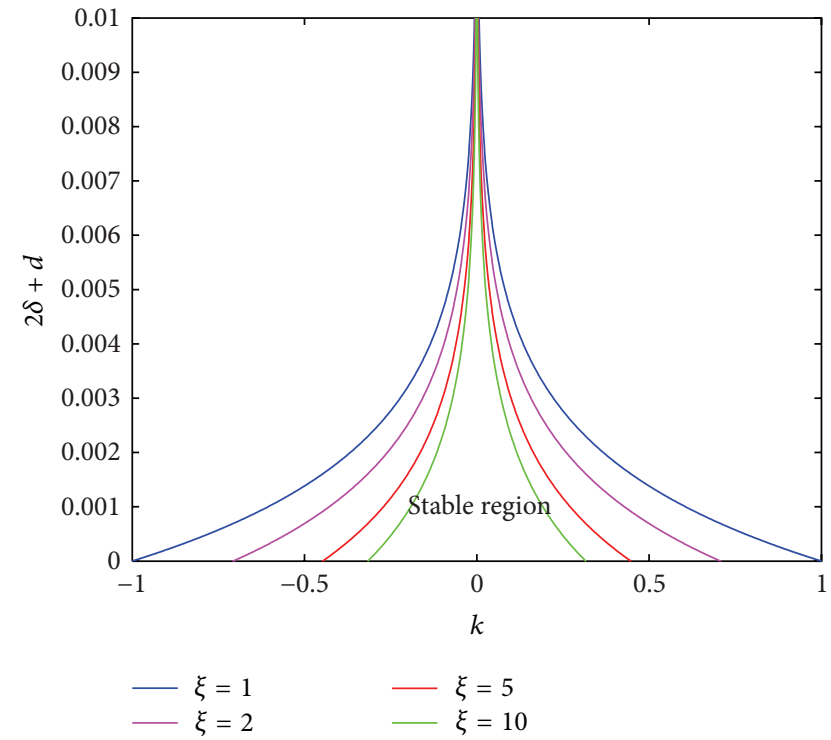

FIGURE 3: The estimation of boundaries of stable region with different $\xi$.

Remark 9. The above theorem gives an estimation of the upper boundary of $2 \delta+d,(2 \delta+d)_{\max }$ :

$$
(2 \delta+d)_{\max }=-\frac{\ln \left(\xi d_{1}\right)}{d_{2}+\varepsilon q+\varepsilon^{-1}} \quad(\xi>1) .
$$

From above we can see that $d_{1}$ should satisfy $0<d_{1}<1$. If $\tau_{k}=\tau_{k}^{l}=\tau_{k}^{r}$, we get the following corollary.

Corollary 10. Le $\lambda_{1}$ be the largest eigenvalue of $\left(I+B^{T}\right)(I+B)$ and $q=\|J\|$. Let $\lambda_{2}$ be the largest eigenvalue of $\left(A+A^{T}\right)$ and impulses equidistant with an interval $\delta>0$. If $0 \leq \lambda_{2}+\varepsilon q+$ $\varepsilon^{-1} \leq-(1 / \delta) \ln \left(\xi \lambda_{1}\right), \xi>1$, then the impulsively controlled memristor-based chaotic circuit system with impulsive time windows (7) at $\tau_{k}=\tau_{k}^{l}=\tau_{k}^{r}$ is asymptotically stable.

Remark 11. If the impulse window $\tau_{k}=\tau_{k}^{l}=\tau_{k}^{r}$, the impulsive time window is reduced into the fixed moments impulse, and Corollary 10 is reduced into Theorem 1 results in [20], which shows that we obtained Theorem 6 is more general.

\section{Numerical Example}

Example 1. We consider the impulsively controlled memristor-based chaotic circuit system with impulsive time windows

$$
\begin{aligned}
& \dot{X}=A X+\phi(X) \quad t \neq \tau_{k}, \tau_{k} \in\left[\tau_{k}^{l}, \tau_{k}^{r}\right), \\
& X\left(\tau_{k}^{+}\right)=B X\left(\tau_{k}^{l}\right) \quad t=\tau_{k}, \\
& X\left(\tau_{0}^{+}\right)=X_{0} \quad k \in N .
\end{aligned}
$$



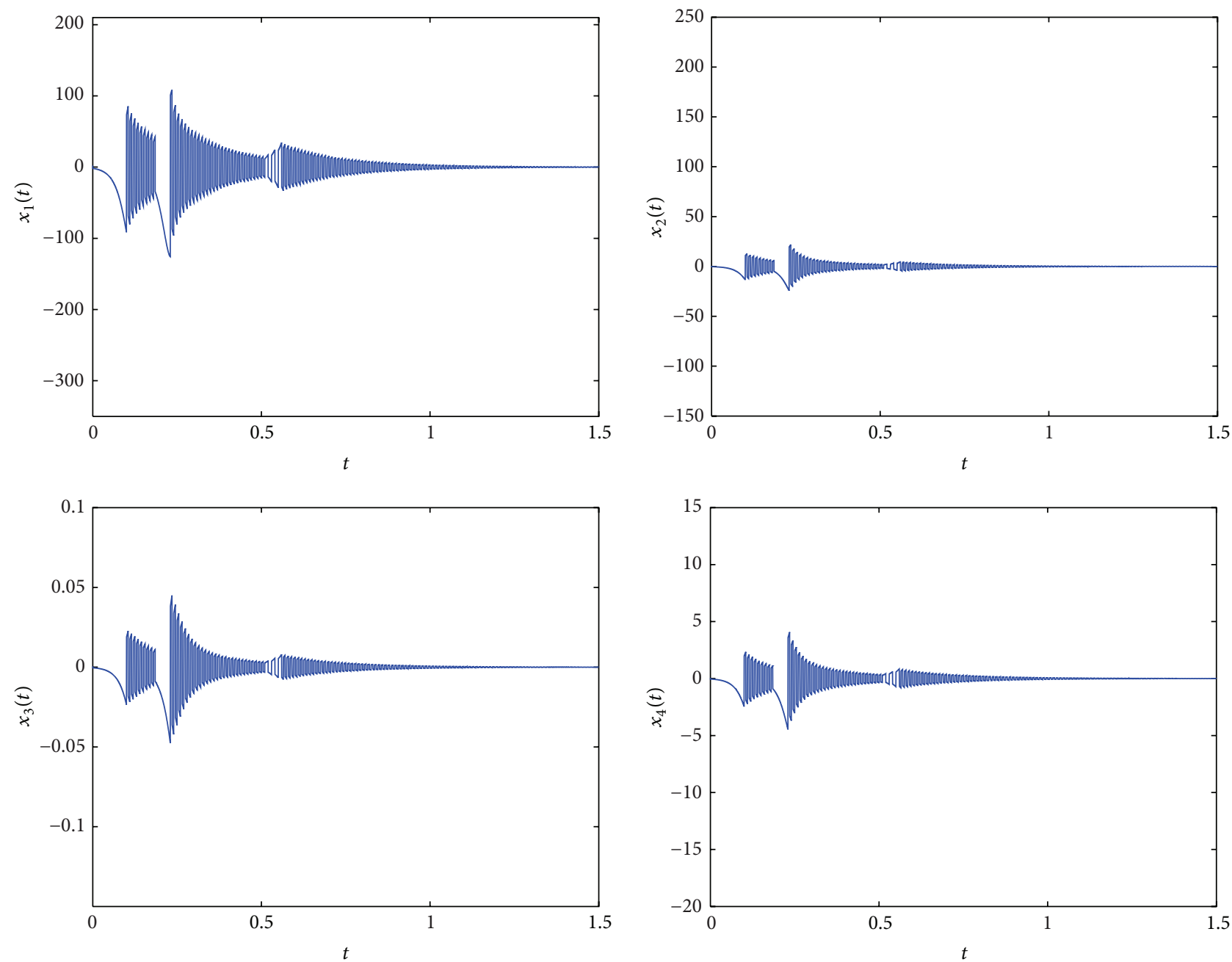

Figure 4: Time response curves of $x_{1}(t), x_{2}(t), x_{3}(t)$, and $x_{4}(t)$ in Example 1.

In this example, we choose the matrix $B$ as

$$
\begin{aligned}
& B=\left(\begin{array}{cccc}
k & 0 & 0 & 0 \\
0 & -1 & 0 & 0 \\
0 & 0 & -1 & 0 \\
0 & 0 & 0 & -1
\end{array}\right) ; \quad \text { then } \\
& B^{T} B=\left(\begin{array}{cccc}
k^{2} & 0 & 0 & 0 \\
0 & 1 & 0 & 0 \\
0 & 0 & 1 & 0 \\
0 & 0 & 0 & 1
\end{array}\right)
\end{aligned}
$$

whose largest eigenvalue is given by $d_{1}=\max \left(1, k^{2}\right)$. Since $d_{1} \in(0,1)$ should be satisfied, we know that $k \in(-1,0) \cup$ $(0,1)$. Let the parameters be $a=1 / 5.6 * 10^{-6}, b=1 / 14.5$; then we have

$$
\begin{aligned}
& A=\left(\begin{array}{cccc}
-\frac{1}{5.6} * 10^{-6} & \frac{1}{5.6} * 10^{-6} & 0 & 0 \\
1 & -1 & -1 & 0 \\
0 & \frac{1}{14.5} & 0 & 0 \\
1 & 0 & 0 & 0
\end{array}\right), \\
& A+A^{T}=\left(\begin{array}{rrrrr}
-3.5714 * 10^{-7} & 1 & 0 & 0 \\
1 & & -2 & -0.9310 & 0 \\
0 & & -0.9310 & 0 & 0 \\
1 & 0 & 0 & 0
\end{array}\right) .
\end{aligned}
$$

We find that $d_{2}=1.2190$; then the stable region for $2 \delta+d$ is $0<2 \delta+d \leq-\frac{\ln \xi+\ln k^{2}}{1.2190+\varepsilon q+\varepsilon^{-1}} \quad k \in(-1,0) \cup(0,1)$. 
Taking the initial condition $\left(x_{1}(0), x_{2}(0), x_{3}(0), x_{4}(0)\right)=$ $(0.1254,0.1402,0.0921,0.0007), \delta=0.005$,

$$
B=\left(\begin{array}{cccc}
-1.8 & 0 & 0 & 0 \\
0 & -1 & 0 & 0 \\
0 & 0 & -1 & 0 \\
0 & 0 & 0 & -1
\end{array}\right)
$$

Figure 3 shows the stable region for different $\xi$. The entire region under the curve $\xi=1$ is the stable region; when $\xi \rightarrow \infty$, the stable region approaches a vertical line $k=0$. Figure 4 shows the stable results within the stable region with $k=-1.8, \delta=0.005$; we conclude that the memristorbased chaotic circuit system with impulsive time windows (7) is asymptotically stable. Figure 4 shows the asymptomatic stability of memristor-based chaotic circuit system with weak control.

\section{Conclusions}

In this paper, we investigated the problem of impulsive control for a class of memristive chaotic systems with impulsive time windows. The new impulsive control strategy where the impulsive effects can occur at a random moment of a determined time interval has been formulated. By using comparison principle, some sufficient conditions which ensure asymptotic stability for the model considered have been obtained. Future research topics include the extension of the results to more high-order memristive systems and delay memristive systems.

\section{Conflict of Interests}

The authors declare that there is no conflict of interests regarding the publication of this paper.

\section{Acknowledgments}

This research is supported by the Natural Science Foundation of China (Grants nos. 61302180 and 61403050) and Postdoctoral Science Foundation of China (Grant no. 2014M562266).

\section{References}

[1] L. O. Chua, "Memristor-the missing circuit element," IEEE Transactions on Circuit Theory, vol. 18, no. 5, pp. 507-519, 1971.

[2] J. M. Tour and T. He, "Electronics: the fourth element," Nature, vol. 453, no. 7191, pp. 42-43, 2008.

[3] D. B. Strukov, G. S. Snider, D. R. Stewart, and R. S. Williams, "The missing memristor found," Nature, vol. 453, pp. 80-83, 2008.

[4] L. O. Chua, M. Itoh, L. Kocarev, and K. Eckert, "Chaos synchronization in Chua's Circuit," Journal of Circuits, Systems and Computers, vol. 3, no. 1, pp. 93-108, 1993.

[5] E. R. Hunt and G. Johnson, "Keeping chaos at bay," IEEE Spectrum, vol. 30, no. 11, pp. 32-39, 1993.

[6] A. I. Panas, T. Yang, and L. O. Chua, "Experimental results of impulsive synchronization between two Chua's circuits,"
International Journal of Bifurcation and Chaos, vol. 8, no. 3, pp. 639-644, 1998.

[7] J. Schweizer and M. P. Kennedy, "Predictive Poincaré control: a control theory for chaotic systems," Physical Review E, vol. 52, no. 5, pp. 4865-4876, 1995.

[8] T. Yang and L. O. Chua, "Impulsive stabilization for control and synchronization of chaotic systems: theory and application to secure communication," IEEE Transactions on Circuits and Systems. I. Fundamental Theory and Applications, vol. 44, no. 10, pp. 976-988, 1997.

[9] T. Yang, L. B. Yang, and C. M. Yang, "Impulsive control of Lorenz system," Physica D: Nonlinear Phenomena, vol. 110, no. 1-2, pp. 18-24, 1997.

[10] T. Yang, Impulsive Systems and Control: Theory and Application, Nova Science Publishers, New York, NY, USA, 2001.

[11] T. Yang, C.-M. Yang, and L.-B. Yang, "Control of Rössler system to periodic motions using impulsive control methods," Physics Letters A, vol. 232, no. 5, pp. 356-361, 1997.

[12] C. D. Li, G. Feng, and T. W. Huang, "On hybrid impulsive and switching neural networks," IEEE Transactions on Systems, Man, and Cybernetics Part B: Cybernetics, vol. 38, no. 6, pp. 1549-1560, 2008.

[13] C. Li, X. Liao, and K.-W. Wong, "Chaotic lag synchronization of coupled time-delayed systems and its applications in secure communication," Physica D. Nonlinear Phenomena, vol. 194, no. 3-4, pp. 187-202, 2004.

[14] Z. Yang and D. Xu, "Stability analysis of delay neural networks with impulsive effects," Dynamics of Continuous, Discretee Impulsive Systems, Series A: Mathematical Analysis, vol. 13, no. 5, pp. 563-573, 2006.

[15] Z. G. Li, C. Y. Wen, and Y. C. Soh, "Analysis and design of impulsive control systems," IEEE Transactions on Automatic Control, vol. 46, no. 6, pp. 894-897, 2001.

[16] J. T. Sun and Y. P. Zhang, "Stability analysis of impulsive control systems," IEE Proceedings: Control Theory and Applications, vol. 150, no. 4, pp. 331-334, 2003.

[17] Y. Liu and S. Zhao, "Controllability for a class of linear timevarying impulsive systems with time delay in control input," IEEE Transactions on Automatic Control, vol. 56, no. 2, pp. 395399, 2011.

[18] X. Wang, C. Li, T. Huang, and X. Pan, "Impulsive control and synchronization of nonlinear system with impulse time window," Nonlinear Dynamics, vol. 78, no. 4, pp. 2837-2845, 2014.

[19] B. Muthuswamy, "Implementing memristor based chaotic circuits," International Journal of Bifurcation and Chaos, vol. 20, no. 5, pp. 1335-1350, 2010.

[20] S. J. Yang, C. D. Li, and T. W. Huang, "Impulsive control and synchronization of memristor-based chaotic circuits," International Journal of Bifurcation and Chaos, vol. 24, no. 12, Article ID 1450162, 12 pages, 2014.

[21] T. Yang, Impulsive Control Theory, Springer, Berlin, Germany, 2001.

[22] I. R. Petersen, "A stabilization algorithm for a class of uncertain linear systems," Systems \& Control Letters, vol. 8, no. 4, pp. 351357, 1987. 


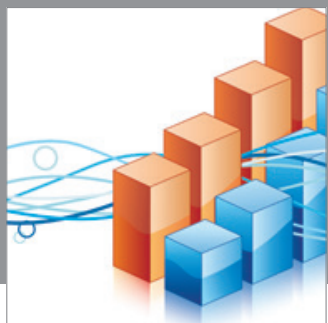

Advances in

Operations Research

mansans

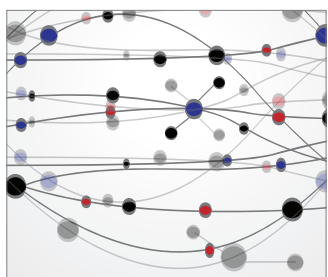

The Scientific World Journal
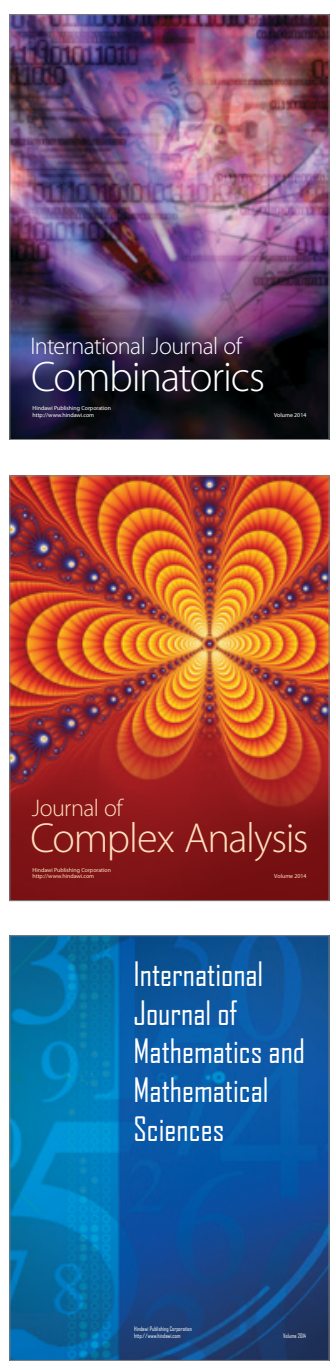
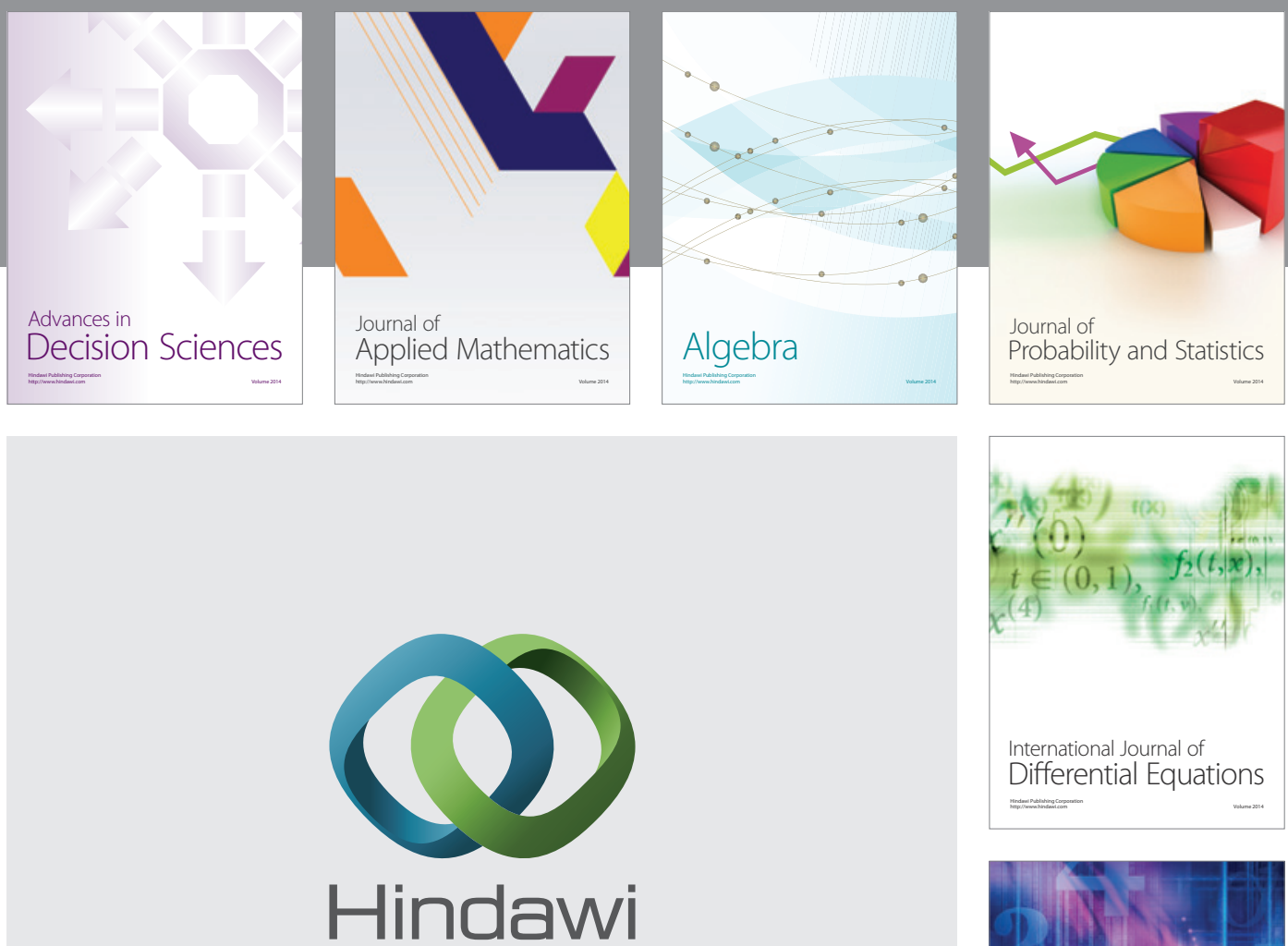

Submit your manuscripts at http://www.hindawi.com
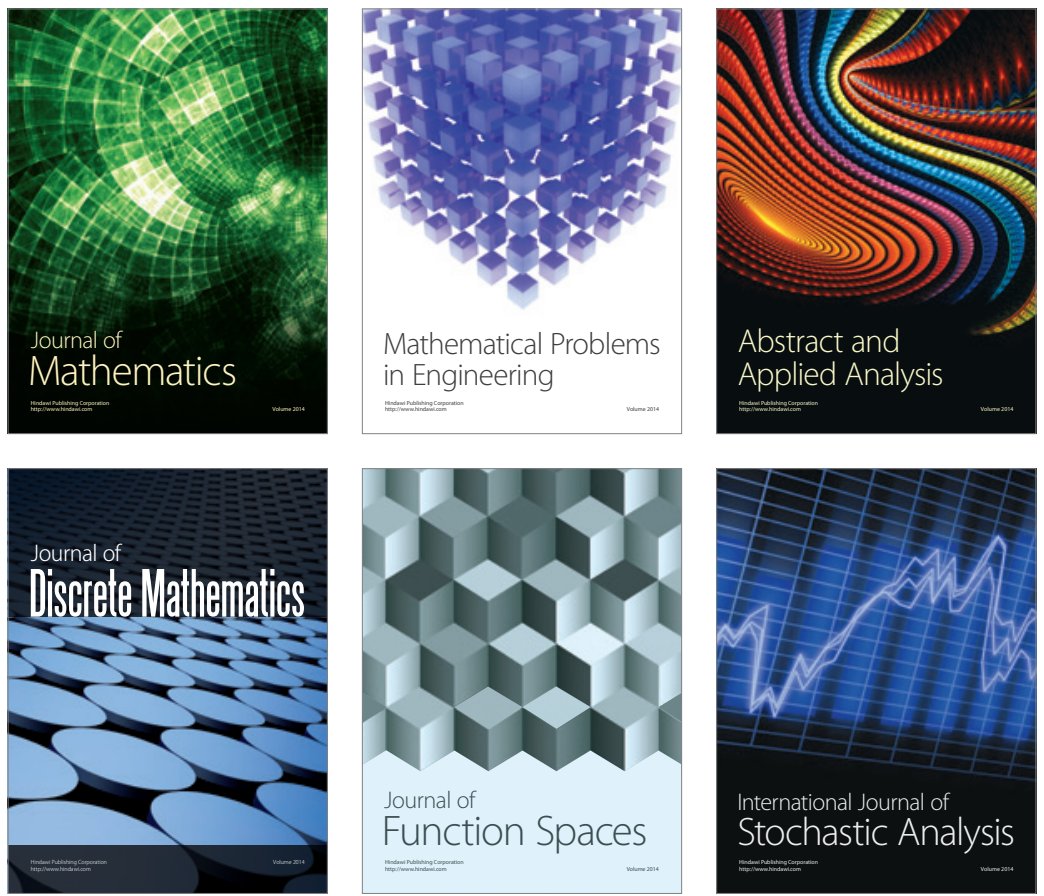

Journal of

Function Spaces

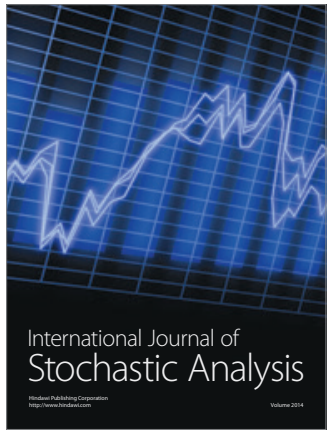

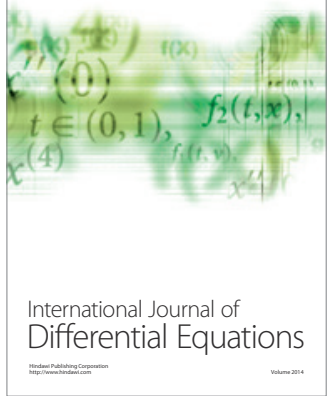
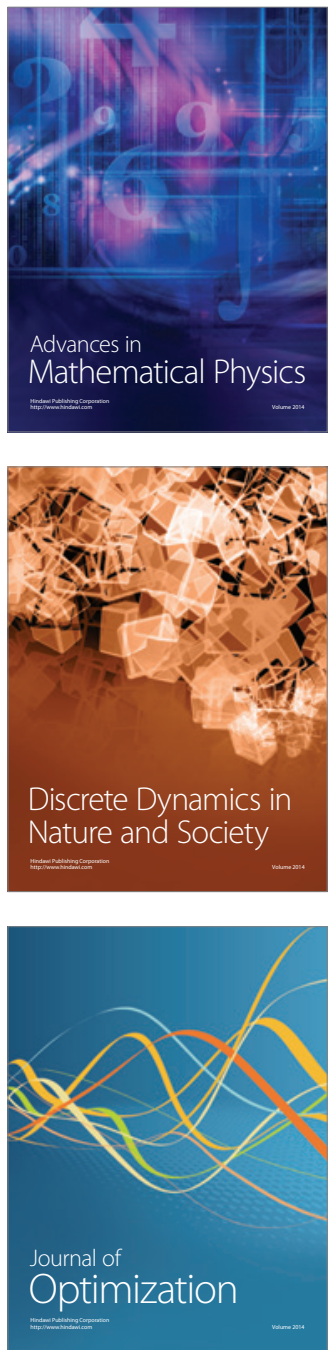